\title{
A TEM-traceable physiologically functional gold nanoprobe that permeates non-endocytic cells
}

This article was published in the following Dove Press journal:

International Journal of Nanomedicine

\author{
Maria Victoria Berberian' \\ Cristian A Pocognoni \\ Luis S Mayorga ${ }^{1,2}$ \\ 'Institute of Histology and \\ Embryology of Mendoza - CONICET, \\ Facultad de Ciencias Exactas y \\ Naturales, Universidad Nacional de \\ Cuyo, Mendoza, Argentina; ${ }^{2}$ Institute \\ of Histology and Embryology of \\ Mendoza - CONICET, Facultad \\ de Ciencias Médicas, Universidad \\ Nacional de Cuyo, Mendoza, Argentina
}

Correspondence: Maria Victoria

Berberian

IHEM - CONICET, Universidad Nacional

de Cuyo, Casilla de correo 56, 5500

Mendoza, Argentina

Tel +54 26I $4494143 ; 7019$

Fax +54 26I 449 4II 7; 7050

Email vberberian@mendoza-conicet.

gob.ar

submit your manuscript

Dovepress of

$4 y$ in 2
Background: Nanoparticles' intracellular fate requires proper internalization. Most cells make use of a battery of internalization pathways, but some are practically sealed, as they lack the biochemical machinery for cellular intake. Non-endocytic cells, such as mammals' spermatozoa, challenge standard drug-delivery strategies.

Purpose: In this article, we present a gold nanoprobe that permeates the external and internal membranes of human sperm.

Methods: Our design makes use of a gold nanoparticle functionalized with a membranepermeable cysteine-rich recombinant protein. The chimeric protein contains two units of physiologically active metallothioneins (MT) that also provide binding motifs to gold and a cell-penetrating-peptide sequence (CPP) that confers cell permeability to the nanoparticle.

Results: Transmission electron microscopy, indirect immunofluorescence, and functional assays show that the nanoprobe is readily internalized in sperm, without compromising cell integrity, while preserving MT's physiological activity. Our findings highlight the potential of CPP-functionalized nanogold for investigating the physiology of otherwise impermeable non-endocytic cells.

Keywords: human sperm, metallothionein, gold nanoparticles functionalization, cell-penetrating peptides, transmission electron microscopy

\section{Introduction}

Gold nanoparticles (AuNPs) have been extensively used for applications in chemistry, biology, and nanotechnology due to their intriguing optical properties. Such properties stem from the way metal valence electrons interact with electromagnetic radiation. At a certain light frequency, the collective oscillation of the electrons produces an intense electromagnetic field on the particle surface that enhances its scattering and lightabsorption capacity. ${ }^{1,2}$ Colloidal gold has also generated great interest as an electrondense non-cytotoxic ${ }^{3}$ and stable cytochemical marker, which is easy to synthesize $e^{4,5}$ and which can be visualized by transmission electron microscopy (TEM) without the need for staining. Several direct and indirect labeling experiments, based on AuNPs, have reported the detection and localization of biochemical targets by TEM, such as antibodies, lectin, and proteins. ${ }^{6,7}$

Thiol groups have the chemical ability to bind to gold surfaces with fairly high affinity, suggesting that proteins bearing a large number of solvent-exposed cysteines could be TEM-localized in cells via association with AuNPs. In this sense, metallothioneins (MTs), which are a family of cysteine-rich (30\%) low-molecular-weight metalloproteins, could be exemplary ligands to gold particles. ${ }^{8,9}$ MTs are ubiquitous in living organisms but, more importantly from the point of view of the present work, they play a role in the maturation of sperm. In general, they are present in most if not all eukaryotic cells and are active within most tissues of higher animals. In particular, 
they have been found in the male mammalian reproductive organs, such as testis, epididymis, prostate, and seminal vesicle. ${ }^{10-13}$ Furthermore, in situ hybridization assays have shown that MTs are regulated during the development of sperm cells, indicating a possible role in male reproduction physiology, ${ }^{14}$ although the specific details of the MTs' roles in gametes functioning are still unclear. ${ }^{13,15}$

Unfortunately, the possibility of investigating the effects of MTs, and other soluble proteins, on sperm physiology is severely limited, as such molecules cannot passively cross cell membranes. Spermatozoa depict a complex and specialized membrane organization and have limited intake and internal transport mechanisms. In particular, the lack of endocytic machinery imposes a very effective biological barrier that makes these cells a difficult target for most drugdelivery strategies.

In this work, we present a gold nanoprobe that is able to translocate the external and internal membranes of, otherwise impermeable, non-endocytic cells. The probe is made of AuNPs coated with a physiologically active cysteine-rich recombinant protein. The protein combines two tandem sequences of the mouse MT, MT1, that provides binding motifs to the TEM-traceable metallic core, with a cell-

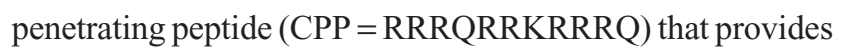
membrane permeation capacity.

The protein that we refer to as CPP-MT also includes His6 and GST tags for detection and purification.

It is well known that CPP translocates biomembranes in a receptor-independent manner and that when conjugated to proteins, DNA, or RNA strands, CPP confers these molecules the ability to diffuse across membranes. ${ }^{16-18}$ In fact, our group has pioneered the use of CPP to design membrane-permeable versions of proteins, such as Rab3A, Rab27 and VPS4A, that have been successfully delivered into human sperm, and allowed to establish their role in the acrosomal exocytosis mechanism. ${ }^{19-21}$ There is also a profuse bibliographic record documenting the cellular uptake of CPP-decorated cargoes, such as macromolecules and NPs. ${ }^{22-26}$ From a mechanistic perspective, the shortcoming of many of these studies is that they have been performed on endocytic cells. This is problematic, as it is then difficult to know whether the cargoes are internalized by endocytosis, by direct translocation of the cell membranes, or following a combination of these two mechanisms.

In this work, we make use of a novel CPP-based design concept that focuses on the various advantages offered by nanogold: 1) TEM and indirect immunofluorescence (IFI) traceability, 2) strong binding to cysteine-rich proteins, $3)$ tunable membrane permeability via appropriate coating, and 4) limited cytotoxicity. We present a detailed investigation of the interaction of our CPP-MT-AuNP probes with living human sperm and analyze their physiological effects. TEM images, IFI, and functional assays show that the nanoprobes are effectively internalized into sperm, while preserving the MT's normal physiological activity, without compromising cell integrity. More in general, our findings highlight the potential of combining AuNPs with chimeric proteins containing cysteine-rich and CPP sequences, for introducing and TEM-tracking active proteins into non-endocytic cells.

\section{Materials and methods \\ Materials}

Human tubal fluid (HTF) medium $(5.94 \mathrm{~g} / \mathrm{L} \mathrm{NaCl}, 0.35 \mathrm{~g} / \mathrm{L}$ $\mathrm{KCl}, 0.05 \mathrm{~g} / \mathrm{L} \mathrm{MgSO}_{4} \cdot 7 \mathrm{H}_{2} \mathrm{O}, 0.05 \mathrm{~g} / \mathrm{L} \mathrm{KH}_{2} \mathrm{PO}_{4}, 0.3 \mathrm{~g} / \mathrm{L} \mathrm{CaCl}_{2}$ $2 \mathrm{H}_{2} \mathrm{O}, 2.1 \mathrm{~g} / \mathrm{L} \mathrm{NaHCO}_{3}, 0.51 \mathrm{~g} / \mathrm{L}$ D-glucose, $0.036 \mathrm{~g} / \mathrm{L} \mathrm{Na}$ pyruvate, $2.39 \mathrm{~g} / \mathrm{L} \mathrm{Na}$ lactate, $0.06 \mathrm{~g} / \mathrm{L}$ penicillin, $0.05 \mathrm{~g} / \mathrm{L}$ streptomycin, $0.01 \mathrm{~g} / \mathrm{L}$ phenol red) was purchased from Irvine Scientific (Santa Ana, CA, USA). Pisum sativum lectin (PSL) labeled with fluorescein isothiocyanate (FITC-PSL), poly-Llysine, bovine serum albumin (BSA), and 4',6-diamidino2-phenylindole were acquired from Sigma-Aldrich S.A. Ni-NTA-agarose was purchased from Qiagen (Tecnolab SA, Buenos Aires, Argentina); DL-dithiothreitol and isopropyl- $\beta$ D-1-thiogalactopyranoside (IPTG) were purchased from ICN (Eurolab SA, Buenos Aires, Argentina). Anti-GST antibody was from Calbiochem (MERCK, Buenos Aires, Argentina). Horseradish peroxidase-coupled anti-rabbit and Cy3-labeled goat anti-rabbit antibodies were from Jackson ImnunoResearch (Seroimmuno Diagnostics, Inc., Tucker, GA, USA). A23187 was from Alomone Labs. Ltd., Jerusalem, Israel. Pelco (Ted Pella Inc., CA, USA) supplied all electron microscopy supplies. All other chemical reagents were of analytical grade and were purchased from ICN or Sigma-Aldrich.

\section{Construction, expression, and purification of permeable MT}

The cDNA encoding a permeable version of MT (CPP-MT) was designed and synthesized by GenScript Corporation for us. The plasmid encoded 1) two tandem sequences of the mouse protein MT-1 (61 AA) that can bind gold; 2) a CPP sequence (RRRQRRKRRRQ) conferring the ability to permeate the cell membrane; and 3) a His6 tag and a GST tag for detection and purification. The protein sequence is presented in Figure 1, in which colored boxes represent different functional moieties.

The plasmid encoding CPP-MT was transferred into Escherichia coli's strain BL21 (DE3) pLysS. Protein expression was induced $2.5-3$ hour at $37^{\circ} \mathrm{C}$ with $1 \mathrm{mM}$ IPTG. Bacteria were harvested and lysed by sonication and 


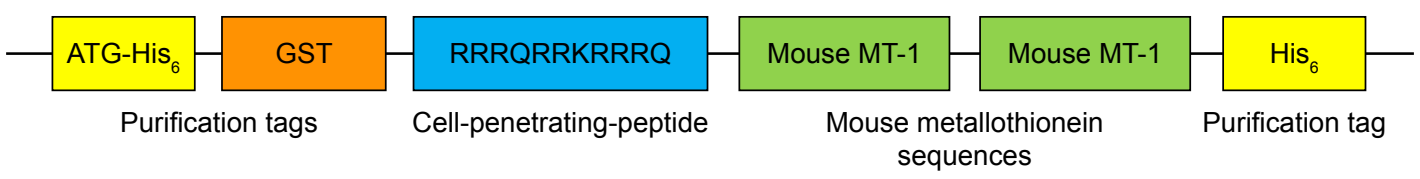

Figure I Schematic representation of the CPP-MT protein.

Notes: The mouse MT-I sequences (green) were fused at their N-terminus to the cell penetrating peptide RRRQRRKRRRQ (blue) to facilitate membrane permeation. Two His ${ }_{6}$ tags (yellow) and a GST tag (orange) were added for affinity purification and immunodetection.

Abbreviations: CPP, cell-penetrating peptide; MT, metallothionein.

the protein was purified under native conditions by affinity chromatography on Ni-NTA-agarose. The CPP-MT isolated in the elution buffer $(250 \mathrm{mM}$ imidazole, $100 \mathrm{mM} \mathrm{NaCl}$, $50 \mathrm{mM}$ Tris-HCl, $\mathrm{pH}$ 8) was passed through a Sephadex G-25 gel filtration column for removing salts and for changing the buffer to PBS ( $\mathrm{pH}$ 7.4). The protein purity was assessed by SDS-PAGE and UV-Vis spectroscopy (See Figures S1 and S2). The concentration was determined by Bio-Rad protein assay in 96-well microplates. BSA was used as a standard, and the results were quantified on a 3550 Microplate Reader (Bio-Rad). After purification, CPP-MT was divided into aliquots and stored at $-80^{\circ} \mathrm{C}$.

Recombinant GST-Rab3A was produced and purified as described in Quevedo et al. ${ }^{27}$

\section{AuNP synthesis, MT conjugation, and characterization}

AuNPs with a diameter of $16.73 \pm 2.78 \mathrm{~nm}$ were prepared by the Turkevich-Frens method. ${ }^{4}$ In this method, $25 \mu \mathrm{L}$ of $20 \%$ (w/v) $\mathrm{HAuCl}_{4}$ (Sigma-Aldrich) aqueous solution was added to $50 \mathrm{~mL}$ of bidistilled water and boiled. Then, $1.6 \mathrm{~mL}$ of $1 \%(\mathrm{w} / \mathrm{v})$ trisodium citrate (Sigma-Aldrich) aqueous solution was added to the solution. Refluxing of the solution continued until the color of the boiling solution changed from colorless to gray-violet and then to red indicating NP formation.

To conjugate the protein to NPs, $100 \mu \mathrm{L}$ of AuNPs (16.73 $\pm 2.78 \mathrm{~nm}$ in diameter, in citrate medium) was brought to $\mathrm{pH} 8.5$ (corresponding to the isoelectric point of the MT) with $\mathrm{NaOH}$. Then, CPP-MT was added to a final concentration of $20 \mu \mathrm{g} / \mathrm{mL}$ and allowed to react at $4^{\circ} \mathrm{C}$ under gentle shaking for 48 hours. This led to the formation of a protein layer bound to the gold surface through a combination of electrostatic interactions and covalent coupling via sulfurgold bonds. ${ }^{7}$ As control, AuNPs were stabilized with $\mathrm{BSA}^{28}$ using the same procedure (BSA final concentration $20 \mu \mathrm{g} / \mathrm{mL}$ at $\mathrm{pH}$ 7.4). Finally, the gold particles were pelleted at $12,000 \mathrm{rpm}$ for 20 minutes to eliminate the citrate buffer and unreacted protein and resuspended in HTF medium $(50 \mu \mathrm{L})$. NP functionalization was monitored by UV-Vis spectroscopy by observing the expected shift on the surface plasmon associated with the formation of the protein cover.
The optimal concentration of CPP-MT was determined experimentally as the minimum amount of protein required to cover the NP surface and prevent flocculation in the presence of $\mathrm{NaCl}$. In brief, $100 \mu \mathrm{L}$ of AuNPs was incubated in Eppendorf tubes with $20 \mu \mathrm{L}$ of CPP-MT at different concentrations $(5-25 \mu \mathrm{g} / \mathrm{mL})$ at $\mathrm{pH}$ 8.5. After 1 hour of incubation at $4^{\circ} \mathrm{C}, 25 \mu \mathrm{L}$ of $10 \%(\mathrm{w} / \mathrm{v}) \mathrm{NaCl}$ was added and the sample was incubated for 15 minutes. The color of the solution was observed and measured spectrophotometrically. Concentrations of CPP-MT lower than $20 \mu \mathrm{g} / \mathrm{mL}$ failed to stabilize AuNPs.

The physicochemical properties of the free AuNPs and CPP-MT-AuNPs were evaluated by TEM, UV-Vis spectroscopy and $\zeta$-potential measurements (see Figure 2 and Table 1). UV-Vis spectra of samples were recorded with a Shimadzu UV-1650 spectrometer within the spectral range of 200-900 nm. NP concentrations were estimated from the UV-Vis spectra as described in Haiss et al. ${ }^{29}$ The zeta-potential was determined by dynamic light scattering, recorded with a DelsaNano C particle analyzer within an electrophoretic cell. Mean values of three consecutive measurements were presented. For TEM of colloidal solutions, a drop was placed on a carbon-coated, formvar-covered copper grid and dried at room temperature (RT). The grid was analyzed using a Zeiss 900 electron microscope, and the diameter of at least 200 particles was measured using ImageJ software to obtain NP size distribution.

\section{Preparation of human sperm samples and coincubation conditions}

Semen samples were provided by masturbation by healthy volunteer donors (age range 21-45) who were free from sexually transmitted diseases. Only samples that met the quality parameters established by the WHO were included in the analysis. Semen was let to liquefy at $37^{\circ} \mathrm{C}$ for $30-60$ minutes. A swim-up protocol was employed to isolate highly motile sperm in $\mathrm{HTF}$ media for 1 hour at $37^{\circ} \mathrm{C}$ in an atmosphere of $5 \% \mathrm{CO}_{2} / 95 \%$ air. Sperm concentration was then adjusted to $10 \times 10^{6}$ cells per milliliter with HTF. In all experiments, cells were incubated in $\mathrm{HTF}$ buffer for 3 hours at $37^{\circ} \mathrm{C}$, which contained proteins CPP-MT and CPP-MT-AuNP or control BSA-AuNP $(20 \mu \mathrm{g} / \mathrm{mL})$. 

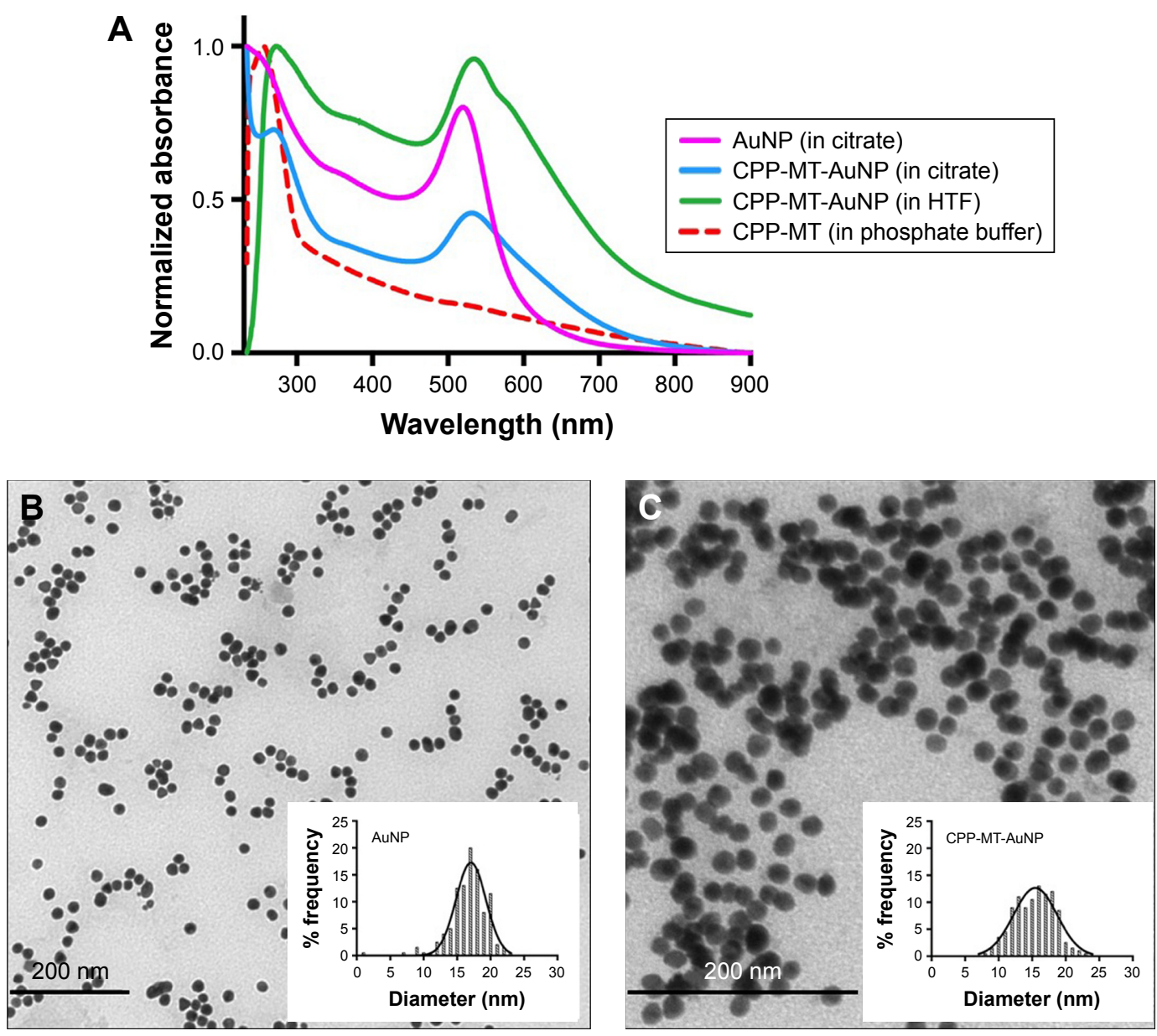

Figure 2 Characterization of gold nanoparticles by SPR spectroscopy and TEM.

Notes: (A) Optical absorption spectra of ligand-free and CPP-MT-conjugated AuNPs in citrate and HTF media. Transmission electron micrographs and particle diameter frequency distribution of ligand-free AuNPs (B) and CPP-MT-conjugated AuNP (C).

Abbreviations: CPP, cell-penetrating peptide; MT, metallothionein; SPR, surface plasmon resonance; TEM, transmission electron microscopy; HTF, human tubal fluid.

\section{Determination of sperm motility, membrane integrity, and morphology}

Motility and membrane integrity were determined according to the WHO Laboratory Manual for the Examination and Processing of Human Sperm criteria (at least 200 cells were scored). ${ }^{30}$ Sperm movements were classified as "progressive (moving actively, either linearly or in a large circle), non-progressive (all other patterns of motility with an absence of progression, eg, swimming in small circles, the flagellar force hardly displacing the head, or when only a flagellar beat can be observed.) or immotile (not moving at all)". Sperm membrane integrity (sperm viability)

Table I Some physicochemical parameters of ligand-free and CPP-MT-conjugated AuNP

\begin{tabular}{|l|l|l|l|}
\hline Gold nanoparticles & UV-Vis $\lambda_{\max }(\mathbf{n m})$ & $\begin{array}{l}\text { DLS, zeta potential } \\
(\mathbf{m V}) \pm \text { SD }\end{array}$ & $\begin{array}{l}\text { TEM, particle diameter } \\
(\mathbf{n m}) \pm \mathbf{S D}^{*}\end{array}$ \\
\hline AuNP in citrate & 520 & $-31.6 \pm 1.5$ & $16.73 \pm 2.78$ \\
\hline CPP-MT-AuNP in citrate & 531 & $-33.9 \pm 0.4$ & - \\
\hline CPP-MT-AuNP in HTF & 534 & $-19.4 \pm 1.7$ & $15.31 \pm 2.94$ \\
\hline BSA-AuNP in citrate & 523 & $-30.8 \pm 1.4$ & - \\
\hline BSA-AuNP in HTF & 523 & $-14.8 \pm 2.3$ & $15.33 \pm 2.39$ \\
\hline
\end{tabular}

Note: *At least 200 NPs were evaluated.

Abbreviations: AuNP, gold nanoparticles; CPP, cell-penetrating peptide; MT, metallothionein; CPP-MT-AuNP, CPP-MT protein-conjugated AuNP; DLS, dynamic light scattering; HTF, human tubal fluid; BSA, bovine serum albumin; TEM, transmission electron microscopy. 
was assessed for treated spermatozoa and controls $(n=4)$ by staining with $0.5 \%$ Eosin Y. Sperm morphology was assessed based on the shape of heads, tails, and midpieces. Two-hundred sperms were examined by both light and TEM microscopy and their shapes recorded and measured using ImageJ software.

\section{IFI assay}

After incubation with the recombinant proteins, CPP-MT, CPP-MT-AuNP, or the non-permeable Rab3A (control), which have a GST-tag, sperm cells were speckled on polyL-lysine-covered slides, fixed in $2 \%$ paraformaldehyde in PBS for 10 minutes at RT, and permeabilized in $0.1 \%$ Triton $\mathrm{X}-100$ in PBS (10 minutes, RT). Cells were incubated in $50 \mathrm{mM}$ glycine-PBS for 30 minutes at RT and then blocked with 5\% BSA in PBS for 1 hour. The sperms were labeled with $20 \mu \mathrm{g} / \mathrm{mL}$ anti-GST antibody (overnight at $4^{\circ} \mathrm{C}$ in $1 \%$ BSA-PBS), followed by Cy3-labeled anti-rabbit IgG as secondary antibodies ( $4 \mu \mathrm{g} / \mathrm{mL}$ in $1 \% \mathrm{BSA}$ ) for 1 hour at $4^{\circ} \mathrm{C}$. To eliminate proteins bound to the outer membrane and to rule out that the translocation of the proteins was a fixation artifact, sperms were treated with trypsin before the fixation as described in Lopez et al. ${ }^{19}$ Finally, cells were fixed ( 1 minute) in cold methanol, stained with FITC-PSL and subsequently washed with distilled water $(20$ minutes $)$ at $4^{\circ} \mathrm{C}$. Slides were mounted in Mowiol. Sperm cells were examined by confocal microscopy using an Olympus FluoView TM FV1000 (Olympus, Argentina), using the FV10-ASW (version 01.07.00.16) software.

\section{Acrosomal exocytosis evaluation}

The acrosomal exocytosis was evaluated by staining with FITC-PSL according to Mendoza et al. ${ }^{31}$ Spermatozoa with intact acrosome show a strong mark with the fluorescent lectin on the acrosome region. Reacted cells show no labeling at the acrosome region, or the labeling is limited to the edge of the granule (equatorial staining). No less than 200 cells were recorded using a Nikon Optiphot II microscope (Nikon, Inc.) equipped with epifluorescence optics. Negative (no stimulation) and positive (stimulated with A23187) controls were included in all experiments. For each experiment, the acrosome exocytosis index was computed by subtracting the number of spontaneously reacted spermatozoa (range $5 \%-15 \%$ ) from all values and by expressing the result as a percentage of the acrosome reaction observed in the positive control (range 25\%-40\%). Experiments showing more than $15 \%$ spontaneously reacted sperm, or less than $10 \%$ response to stimulation, were excluded from the analysis.
TEM assays of the coincubation of CPPMT-AuNP (or BSA-AuNP) with sperm

We processed human spermatozoa $\left(10 \times 10^{6}\right.$ cells $\left./ \mathrm{mL}\right)$ as described earlier. Then, cells were fixed at RT with $2.5 \%$ $(\mathrm{w} / \mathrm{v})$ glutaraldehyde in phosphate buffer. Fixed samples were washed twice in PBS and post-fixed in $1 \%(\mathrm{w} / \mathrm{v})$ osmium tetroxide-PBS for 1 hour at RT, washed three times in PBS, and dehydrated with increasing concentrations of cold acetone. Cells were infiltrated at RT in 1:1 acetone, spurred for 2 hours or overnight, and finally embedded in fresh resin overnight. Samples were cured 24 hours at $70^{\circ} \mathrm{C}$. A diamond knife (Diatome) was used to cut thin sections $(80 \mathrm{~nm})$ on a Leica Ultracut R ultramicrotome. To avoid spurious signals, the samples were not stained with uranyl acetate/lead citrate. TEM grids were photographed with a Zeiss 900 electron microscope at $80 \mathrm{kV}$. Representative images were selected for the manuscript.

\section{Ethics approval and informed consent}

We are cognizant of the Argentinean (ANMAT 5330/97) and international (Declaration of Helsinki) principles and bioethical codes and guarantee that all procedures carried out in this study were in compliance with both. Human subjects were involved in this project for the purpose of semen donation. The subject population consisted of healthy male donors who were 21 years of age or over. All donors signed a written informed consent form at the time of their enrollment. The Bioethical Committee of the Medical School of the National University of Cuyo, Mendoza, approved our protocol for the collection and manipulation of human sperm samples. All laboratory procedures followed the safety regulations of the Medical School.

\section{Results and discussion Characterization of AuNP and AuNP-labeled CPP-MT}

Monodisperse spherical AuNPs were synthesized by the Turkevich-Frens method ${ }^{4}$ and subsequently conjugated to the active cysteine-rich recombinant CPP-MT protein. As stated in the introduction, the protein $(43 \mathrm{kDa})$ combines two tandem sequences of the mouse MT (MT1), a CPP sequence $(C P P=R R R Q R R K R R R Q)$ conferring the ability to permeate cell membrane, plus His6 and GST tags for detection and purification. 
To confirm functionalization of AuNPs with CPP-MT, ligand-free and conjugated gold particles were characterized using UV-Vis spectrophotometry. Due to surface plasmon resonance (SPR), the UV-Vis spectra of ligand-free AuNPs exhibited a distinct extinction peak at $520 \mathrm{~nm}$ wavelength, while the AuNP covalently bonded to CPP-MT exhibited the plasmon band shifted to $531 \mathrm{~nm}$ (Figure 2A and Table 1). The red shift observed in the UV-Vis spectra of CPP-MT-AuNP was expected, as the SPR band intensity and wavelength are modulated by all those factors that affect the particle surface charge density, such as particle size, shape, state of aggregation, surface modifications, and the dielectric constant of the solvent. ${ }^{32}$

Furthermore, particle diameters were $16.73 \pm 2.78 \mathrm{~nm}$ for ligand-free AuNPs, 15.31 $\pm 2.94 \mathrm{~nm}$ for CPP-MT-AuNPs, and 15.33 $\pm 2.39 \mathrm{~nm}$ for BSA-coated AuNPs (Table 1). The particle size distribution measured by TEM is plotted on the histograms shown in Figure 2B and C.

Protein conjugation to AuNP was also assessed by zeta potential measurements (Table 1), revealing that non-conjugated AuNPs were electrostatically stabilized in citrate solution by a zeta potential of $-31.6 \pm 1.5 \mathrm{mV}$. The zeta potential of CPP-MT-AuNP in a similar solution was evaluated to be slightly more negative $-33.9 \pm 0.4 \mathrm{mV}$, due to the additional charge provided by the CPP-MT, which resulted in additional electrosteric stabilization of the colloid. The zeta potential of the BSA-coated-AuNP control, in citrate medium, was $-30.8 \pm 1.4 \mathrm{mV}$. After transferring the conjugated CPP-MT-AuNP and BSA-AuNP to the HTF medium, a significant decrease in zeta potential was observed for both colloidal dispersions $(-19.4 \pm 1.7$ and $-14.8 \pm 2.3 \mathrm{mV}$ respectively, Table 1). Although the low values suggest that the stability should decrease, such values are within the boundaries that define stable colloidal dispersions. In fact, the two HTF preparations showed excellent long-term stability and no signs of particle aggregation. Likely, such long-term stability can be attributed to steric stabilization by the CPP-MT and BSA protein coronas, thereby preventing particle aggregation.

\section{Evaluation of sperm motility, membrane integrity, and morphology after coincubation with CPP-MT-AuNP or CPP-MT}

In order to evaluate the possible cytotoxic effects of the gold nanoprobe on human sperm, cells were examined after 3 hours of coincubation. Some of the viability parameters recommended by the $\mathrm{WHO}$, such as motility, membrane integrity, and overall morphology, were assessed by optical microscopy. Moreover, sperm morphology was also assessed by TEM in order to analyze the internal structure and organelles. The effects induced by the nanoprobes were always compared with those of the gold-free CPP-MT soluble protein.

The analysis of sperm morphology included the assessment of cells heads, midpieces, and tails. After CPP-MT-AuNP or CPP-MT exposure, we did not find any abnormal morphological changes in the size and shape of the cells, such as detached heads and coiled, rolled, or bent tails. Moreover, TEM inspection of the cells' ultrastructure did not reveal any obvious anomalies. Cells membrane integrity (Figure 3A) and motility (Figure 3B)
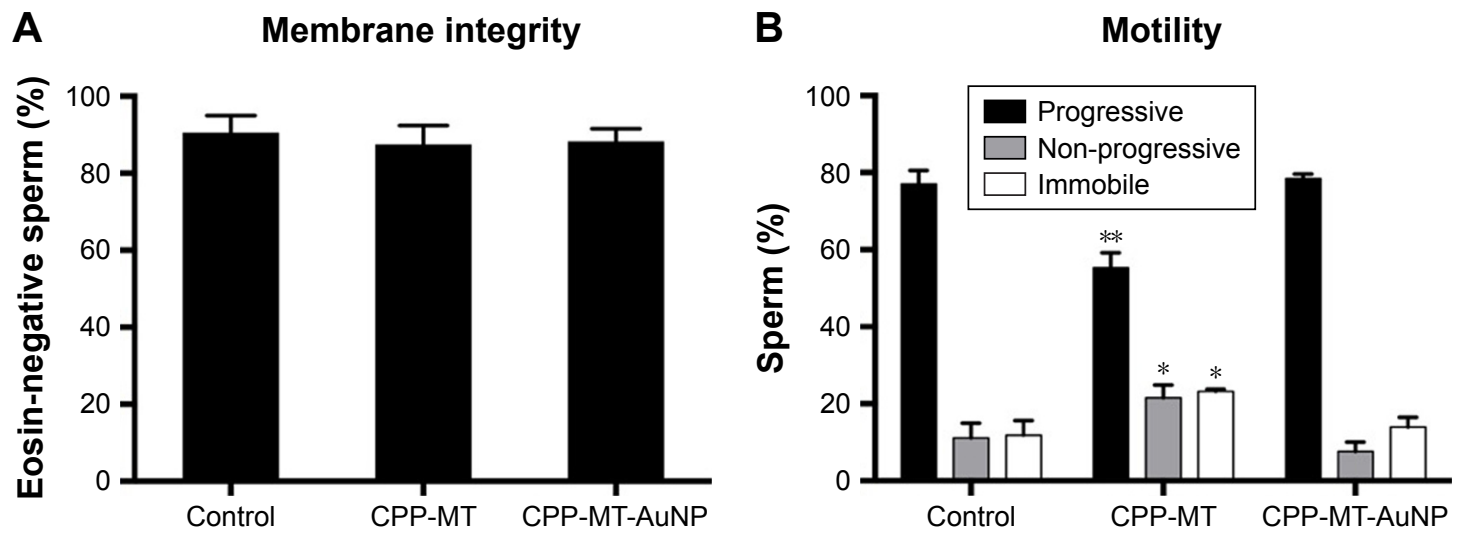

Figure 3 Vitality parameters of untreated spermatozoa (control) and after coincubation with CPP-MT or CPP-MT-AuNPs.

Notes: Sperm were incubated with $20 \mu \mathrm{g} / \mathrm{mL}$ of CPP-MT or CPP-MT-AuNP for 3 hours at $37^{\circ} \mathrm{C}$ in HTF medium. (A) Sperm membrane integrity. (B) Sperm motility. Black bars: progressive motility; gray bars: non-progressive motility; white bars: immobile sperm. The data represent mean values \pm SEM, calculated from three independent experiments in which at least 200 cells were quantified. One-way ANOVA showed no statistically significant difference between relative membrane integrity percentages of all analyzed samples (A). In (B) only CPP-MT samples show statistically significant difference versus the control group, $* *(P<0.00 \mathrm{I})$ and $*(P<0.05)(D u n n e t t ' s t$-test). Abbreviations: AuNP, gold nanoparticles; CPP, cell-penetrating peptide; MT, metallothionein; CPP-MT-AuNP, CPP-MT protein-conjugated AuNP. 
also remained unaffected after CPP-MT-AuNP exposure (Figure 3A), thereby suggesting that the CPP-MT-AuNP probes have no effect on standard viability parameters of human sperm. Interestingly, exposure of cells to the CPP-MT protein showed a slight but significant decrease in sperm motility in comparison to untreated or CPP-MT-AuNPtreated sperm (Figure 3B). This observation suggests that the binding of CPP-MT to AuNPs alleviates, probably due to concentration moderation effects, the negative impact of the protein on the sperm's motility. It is reasonable to speculate that the gold particle would slow down the translocation of CPP-MT-AuNP and decrease concentration-dependent deleterious effects on the cells.

\section{IFI assays after incubation with CPP-MT-} AuNP or CPP-MT

In order to test the efficiency of the CPP to promote the permeation of MT (which has a GST-tag) into spermatozoa, IFI assays with an anti-GST-antibody were performed after 3 hours of coincubation at $37^{\circ} \mathrm{C}$. As a negative control, we used a recombinant non-permeable Rab3A protein, which also had a GST-tag. Rab3A is similar in size to CPP-MT, plays a central role in sperm acrosomal exocytosis, and has been extensively investigated in our laboratory. In order to rule out that the translocation of the proteins was a fixation artifact, or that proteins were stuck to the outer cell membrane, cells were treated with trypsin before fixation. ${ }^{19}$ Under such conditions, GST-Rab3A was not detected by immunofluorescence (Figure 4), whereas the exogenous recombinant
A

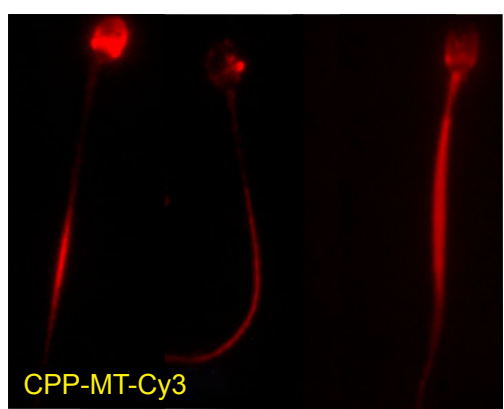

B

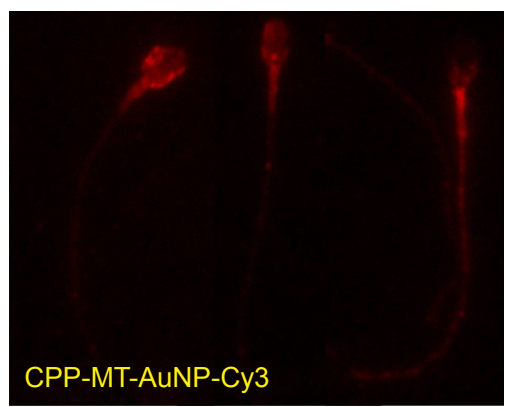

C

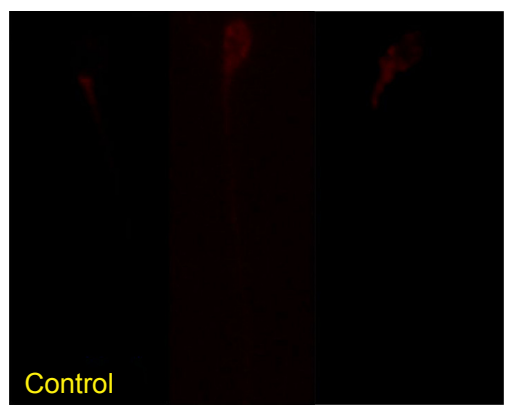

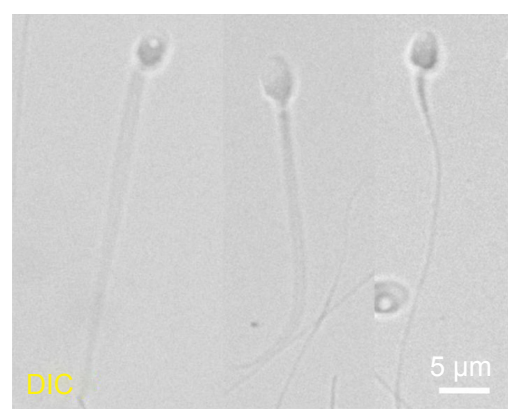
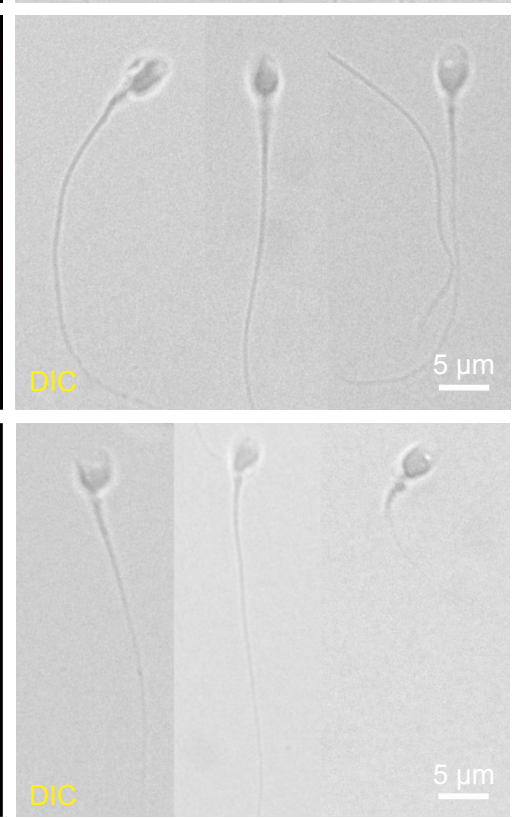
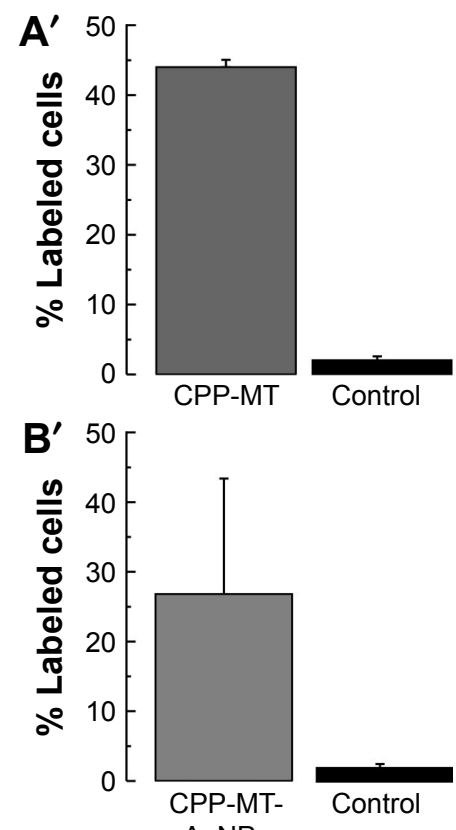

AuNPs
Control

Figure 4 CPP-MT and CPP-MT-AuNP translocate intact human sperm.

Notes: Sperm were incubated with $20 \mu \mathrm{g} / \mathrm{mL}$ of CPP-MT (A), CPP-MT-AuNP (B), or non-permeable GST-Rab3A (C, control) for 3 hours at $37^{\circ} \mathrm{C}$ in HTF medium. Afterward, cells were treated with trypsin to eliminate external probe. Then, cells were fixed and spotted on glass slides and labeled with Cy3-coupled anti-GST antibody. At least 100 cells were quantified, and the population of sperm showing positive label was recorded. The bars represent mean values \pm SEM, calculated from three independent experiments. The images are representative of the experimental conditions described. One-way ANOVA shows statistically significant difference between relative labeled cells percentages incubated with CPP-MT ( $\left.\mathbf{A}^{\prime}\right)$ or CPP-MT-AuNP $\left(\mathbf{B}^{\prime}\right)$ vs the control group, $P<0.000$ I (Dunnett's $t$-test).

Abbreviations: AuNP, gold nanoparticles; CPP, cell-penetrating peptide; MT, metallothionein; CPP-MT-AuNP, CPP-MT protein-conjugated AuNP; HTF, human tubal fluid. 
protein CPP-MT was detected by immunofluorescence in whole cells (head, middle, and principal pieces), indicating that the recombinant protein translocates the cell membrane (Figure 4A). Subsequently, we tested the behavior of the CPP-MT-AuNP probe, which depicted a similar staining pattern to the free CPP-MT protein (Figure 4B). The fluorescent mark was detected on the entire head (cytoplasm, nucleus, and acrosome), the middle, and the principal pieces. In general, $40 \%-45 \%$ of the cells exhibited CPP-MT or CPP-MT-AuNP staining (Figure 4A and B).

The previous observations are remarkable as they show that the CPP confers permeability both to the $43 \mathrm{kDa}$ MT and the AuNP attached to them. Clearly, the insertion of a single CPP sequence within the primary structure of large gold-binding protein facilitates the delivery of the proteinloaded nanoprobe to the sperm cytoplasm. Moreover, the fact that both CPP-MT and CPP-MT-NP traverse the cell membranes strongly suggests that the CPP sequence is exposed to solution. This makes chemical sense, as arginine and lysine, which make most the CPP sequence, are expected to be positively charged at $\mathrm{pH} \sim 7.0$. Thus, the binding of CPP-MT and CPP-MT-NP to the cell surface is expected to be driven by electrostatic forces, as supported by a large body of bibliography on CPP internalization mechanisms. ${ }^{33,34}$ Notice that the CPP preserves its cell permeation activity even when acting as a linker between other protein moieties, as reported in previous references. ${ }^{19,21,35}$

\section{TEM visualization of membrane- translocated probes}

The high-electron density of AuNPs makes them clearly visible in biological samples by TEM. TEM and IFI are indeed complementary techniques, but the former is more direct and accurate than the latter at the time of quantifying the population of cell-internalized probes. In TEM, the analysis is done by explicitly counting the particles located within the boundaries of intact cells. In IFI, probes internalization is inferred from the amount of fluorescent antibody bound to the target protein. In addition, TEM allows the examination of internal cellular structures and provides reliable information on the exact location and internal distribution of AuNP. For example, by TEM, it is possible to examine the association with the structural elements that make the three main segments of sperm: head, middle, and principal pieces. The head contains only a minimal cytoplasm and the nucleus, with densely coiled chromatin fibers and surrounded by a large acrosomal vesicle. The granule's membranes overlying the nucleus and underlying the plasma membrane are called the "inner" and "outer" acrosomal membranes, respectively. The middle piece contains the sperm centriole and the central filamentous core with plenty of mitochondria spiraled around it. The principal piece or tail has a sheath of ring fibers around the axoneme.

A detailed TEM study confirmed the cellular translocation of CPP-MT-AuNP, which is in agreement with the results described in the previous section, and it allowed the intracellular fate of the nanoprobe to be identified. The CPP-MTfunctionalized particles translocated the cell membrane after 3 hours of incubation, without disrupting the cell's internal structure and organelles. Non-aggregated AuNPs were visualized inside the sperm head, middle, and principal pieces. In fact, aggregates were mostly localized in the extracellular space, near the cell's outer membrane (Figure 5). Particles spotted in the head region were mostly located in the cytosol and the acrosome, while only a few particles were observed inside the nucleus. In contrast, almost no particles were found within cells treated with BSA-coated AuNP (control). The images shown in Figure 5 were selected from a large number of observations performed during the course of four separate experiments. It is also important to mention that in order to avoid spurious signals, in these experiments, samples were not stained with heavy-metal salts (lead citrate and uranyl acetate), as is normally done to enhance contrast in TEM images.

In sum, our results indicate that the CPP-MT-AuNP probe is a functioning tool that can be safely delivered and TEM-tracked into non-endocytic cells. Although other authors have reported the use of MTs labeled with gold (metal or gold salts) as clonable tags for TEM, no one has proposed a tag like the one developed this study. ${ }^{36-39}$ Our tag is cell permeable and tightly binds to sizable AuNPs. Moreover, this new technique offers a simple alternative to complex immunostaining with colloidal gold, as traditionally used to localize proteins by TEM. Our method eliminates the need for primary and secondary antibodies and allows a much more accurate localization of the functional protein since the AuNP binds to the CPP-MT at a short distance (1-2 nm). In immunogold techniques, the AuNP is around 15-30 nm away from the site to which the primary antibody is bound. For example, gold immuno-TEM does not allow the determination of whether a protein is associated to the outer acrosomal membrane, the plasma membrane, or both, since the label is generally spread over a wider band, so specific localization is ambiguous.

\section{CPP-MT-AuNP inhibits acrosomal exocytosis in human sperm}

Acrosomal exocytosis in mammalian sperm is a biochemically regulated secretion process, which is a definite 

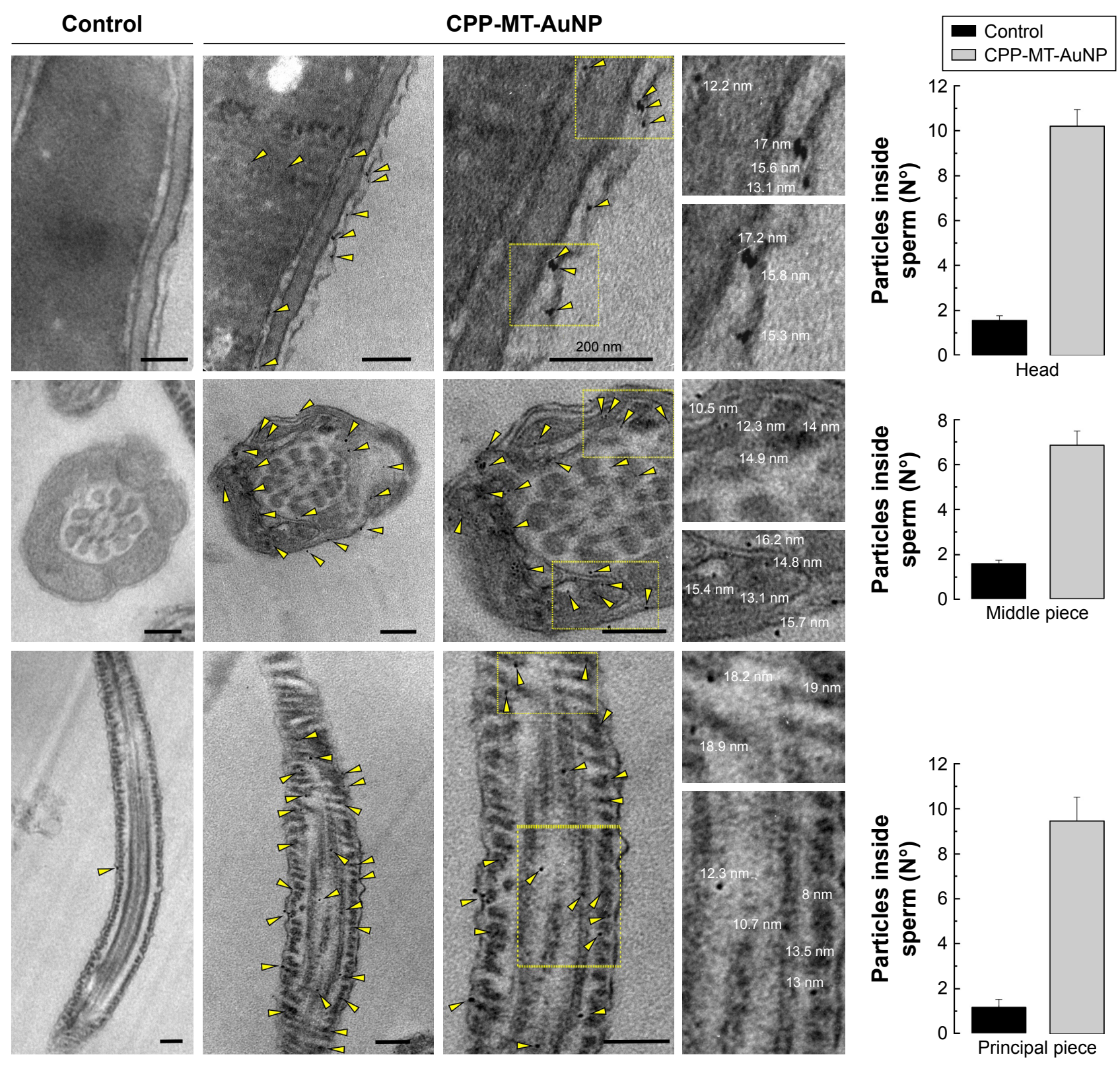

Figure 5 Internalized CPP-MT-AuNP complexes can be found in various human sperm intracellular structures.

Notes: Sperm were incubated with $20 \mu \mathrm{g} / \mathrm{mL}$ CPP-MT-AuNP or BSA-coated AuNP (control) for 3 hours. Samples were fixed and processed for electron microscopy as described in section "Materials and methods". Left: TEM image gallery showing representative AuNPs localization for each experimental condition. Right: the number of incorporated AuNPs was quantified for each sperm region (head, middle, and principal pieces). Scale bars $=200 \mathrm{~nm}$. The data represent mean values \pm SEM, calculated from four independent experiments with at least 100 cells analyzed. One-way ANOVA shows statistically significant difference between particles inside sperm incubated with CPP-MT-AuNP vs the control group for all the samples analyzed, $P<0.000$ I (Dunnett's $t$-test).

Abbreviations: AuNP, gold nanoparticles; CPP, cell-penetrating peptide; MT, metallothionein; CPP-MT-AuNP, CPP-MT protein-conjugated AuNP; BSA, bovine serum albumin; TEM, transmission electron microscopy.

requirement for physiological fertilization. The sperm head contains a large secretory granule, the acrosome, overlying the sperm's nucleus. During acrosomal exocytosis, the content of this granule is released and enzymes facilitate the passage of the sperm through the zona pellucida of the egg. As some of us have recently reported, membrane-impermeable MT inhibits acrosomal exocytosis in permeabilized sperm and interacts specifically with GTP-bound ADP-ribosylation factor $6 .{ }^{40}$
First, in order to assess whether the chimeric membranepermeable CPP-MT was functionally active on its own, we performed standard exocytic assays under physiological conditions. The protein was coincubated with the cells at different concentrations $(0.5-2 \mu \mathrm{M})$ and its effects were monitored by following the calcium-ionophore-triggered (A23187) acrosomal exocytosis process. ${ }^{41}$ The permeable CPP-MT successfully inhibited exocytosis in a dose-dependent manner, confirming not only that the protein was internalized but that 

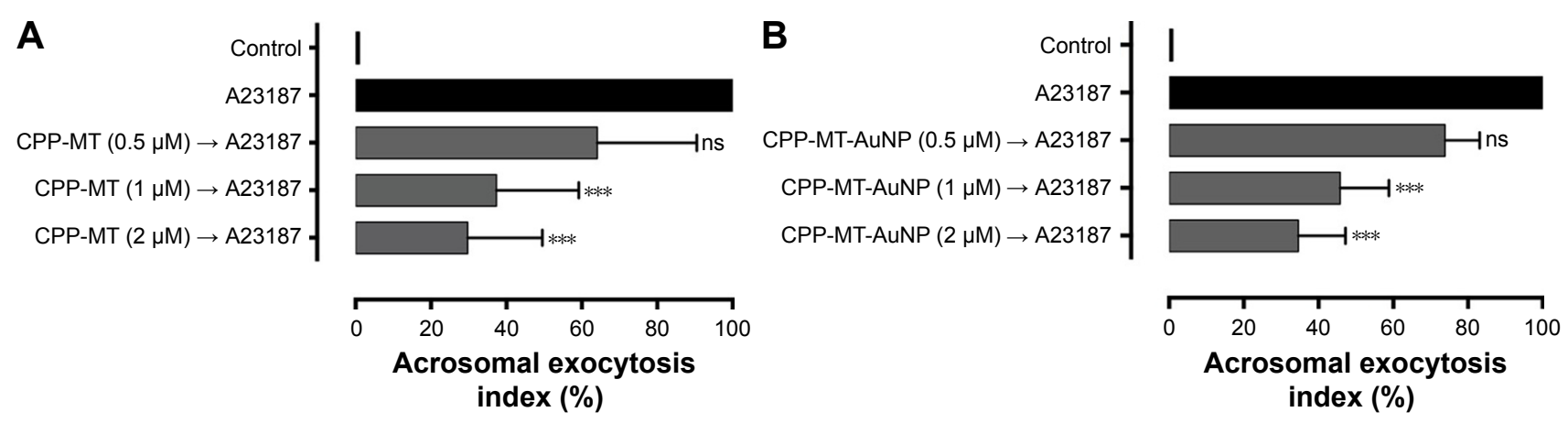

Figure 6 Membrane-permeable CPP-MT and CPP-MT-AuNP inhibit acrosomal exocytosis in sperm.

Notes: Human sperm were incubated for 1 hour at $37^{\circ} \mathrm{C}$ in HTF medium with increasing concentrations of recombinant CPP-MT unconjugated (A) or conjugated to AuNPs (B). Afterward, the cells were incubated for 15 minutes without stimulus (control) or with $10 \mu$ M A23I87. After incubation, the samples were fixed and the acrosomal exocytosis index was evaluated using PSL-FITC and calculated as detailed in section "Materials and methods". The data represent mean values \pm SEM and were calculated from three independent experiments. Two-way ANOVA shows statistically significant difference $(* * * P<0.000 \mathrm{I})$ and nonsignificant difference (ns; $P>0.0 \mathrm{I})$ compared with A23I 87 (Dunnett's $t$-test).

Abbreviations: AuNP, gold nanoparticles; CPP, cell-penetrating peptide; MT, metallothionein; CPP-MT-AuNP, CPP-MT protein-conjugated AuNP; HTF, human tubal fluid.

it also kept its native functionality (Figure 6A). Second, and more importantly, in order to determine whether the protein activity was affected upon binding to AuNP, the nanoprobe CPP-MT-AuNP was coincubated with sperm and exocytic assays were repeated. Effectively, our results (Figure 6B) showed that the presence of the gold nanocore preserves the MT's native activity, since we still observed dose-dependent inhibition of acromosomal exocytosis, suggesting that the protein is not inactivated upon binding to the gold particles. These observations are particularly relevant, as the role of MT on sperm physiology is largely unknown, and the present CPP-MT-AuNP probe may offer distinctive advantages to design MT purpose-specific experiments.

\section{Conclusion}

The delivery of NPs and membrane-impermeable macromolecules into the cytoplasm of non-endocytic cells is severely hindered by the lack of active cellular intake mechanisms. Such conditions impose a serious technical challenge when investigating, for example, the cell biology of mammals' gametes. Cargo delivery strategies employed for these cells are case specific, and they rarely tackle the problem of cargo traceability. Evidently, finding a general solution to such a hurdle is desirable as it may speed up progress in the cell biology of reproduction and other fields.

In the previous sections, we have discussed a gold nanoprobe that permeates the external and internal membranes of human sperm. The probe is made of AuNPs covered with a recombinant protein. The protein merges two sequences of MT with a CPP. At variance with previously published work, ${ }^{42-45}$ our CPP-MT-AuNP probe translocates the sperm membranes, while preserving the MTs' physiological activity. In the absence of explicit structural information, the fact that the protein's function is preserved upon cellular uptake suggests that the structure of the MT moieties is not severely affected upon binding to AuNP. The probe does not compromise cell integrity and shows low levels of cytotoxicity as measured by standard cell viability parameters. Moreover, our electron-dense probe is easily traceable by TEM, can be identified by IFI, and sensed by complementary functional assays, thus offering the distinctive possibility of correlating space localization with triggered physiological response. More in general, the probe design concept may become useful for introducing and visualizing other biologically active proteins into non-endocytic cells, providing a simpler alternative to the traditional complex immunostaining with colloidal gold for TEM localization of proteins.

\section{Acknowledgments}

The authors thank M Furlan and E Bocanegra for technical assistance. This work was supported by Agencia Nacional de Promoción Científica y Tecnológica (ANPCyT), Argentina (grants PICT-2013-1433 and PICT-2016-0894) and the Secretaria de Ciencia y Técnica de la Universidad Nacional de Cuyo (SeCyT), Mendoza, Argentina (grants M002 and M024 SeCyT-UNCUYO).

\section{Disclosure}

The authors report no conflicts of interest in this work.

\section{References}

1. Link S, El-Sayed MA. Spectral properties and relaxation dynamics of surface plasmon electronic oscillations in gold and silver nanodots and nanorods. J Phys Chem B. 1999;103(40):8410-8426. 
2. Link S, El-Sayed MA. Optical properties and ultrafast dynamics of metallic nanocrystals. Annu Rev Phys Chem. 2003;54:331-366.

3. Moretti E, Terzuoli G, Renieri T, et al. In vitro effect of gold and silver nanoparticles on human spermatozoa. Andrologia. 2013;45(6):392-396.

4. Turkevich J, Stevenson PC, Hillier J. A study of the nucleation and growth processes in the synthesis of colloidal gold. Discuss Faraday Soc. 1951;11:55-75.

5. He H, Xie C, Ren J. Nonbleaching fluorescence of gold nanoparticles and its applications in cancer cell imaging. Anal Chem 2008;80(15):5951-5957.

6. Goodman SL, Hodges GM, Trejdosiewicz LK, Livingston DC. Colloidal gold markers and probes for routine application in microscopy. J Microsc. 1981;123(Pt 2):201-213.

7. Boisselier E, Astruc D. Gold nanoparticles in nanomedicine: preparations, imaging, diagnostics, therapies and toxicity. Chem Soc Rev. 2009;38(6):1759-1782.

8. Coyle P, Philcox JC, Carey LC, Rofe AM. Metallothionein: the multipurpose protein. Cell Mol Life Sci. 2002;59(4):627-647.

9. Vasák M. Advances in metallothionein structure and functions. J Trace Elem Med Biol. 2005;19(1):13-17.

10. Nishimura H, Nishimura N, Tohyama C. Localization of metallothionein in the genital organs of the male rat. $J$ Histochem Cytochem. 1990;38(7):927-933.

11. Suzuki T, Yamanaka H, Tamura Y, et al. Metallothionein of prostatic tissues and fluids in rats and humans. Tohoku J Exp Med. 1992; 166(2):251-257.

12. Suzuki T, Yamanaka H, Suzuki K, et al. Immunohistochemical demonstration of metallothionein in the rat epididymis and spermatic cord Zool Sci. 1992;9:1009-1015.

13. Elgazar V, Razanov V, Stoltenberg M, et al. Zinc-regulating proteins, ZnT-1, and metallothionein I/II are present in different cell populations in the mouse testis. J Histochem Cytochem. 2005;53(7):905-912.

14. Tohyama C, Nishimura N, Suzuki JS, Karasawa M, Nishimura H. Metallothionein mRNA in the testis and prostate of the rat detected by digoxigenin-labeled riboprobe. Histochemistry. 1994;101(5): 341-346.

15. Suzuki T, Nakajima K, Yamamoto A, Yamanaka H. Metallothionein binding zinc inhibits nuclear chromatin decondensation of human spermatozoa. Andrologia. 1995;27(3):161-164.

16. Koren E, Torchilin VP. Cell-penetrating peptides: breaking through to the other side. Trends Mol Med. 2012;18(7):385-393.

17. Copolovici DM, Langel K, Eriste E, Langel Ü. Cell-penetrating peptides: design, synthesis, and applications. ACS Nano. 2014, 8(3):1972-1994.

18. Dinca A, Chien WM, Chin MT, Chinint M. Intracellular delivery of proteins with cell-penetrating peptides for therapeutic uses in human disease. Int J Mol Sci. 2016;17(2):263-276.

19. Lopez CI, Belmonte SA, de Blas GA, Mayorga LS. Membrane-permeant Rab3A triggers acrosomal exocytosis in living human sperm. Faseb $J$. 2007;21(14):4121-4130.

20. Bustos MA, Lucchesi O, Ruete MC, Tomes CN. Membrane-permeable Rab27A is a regulator of the acrosome reaction: Role of geranylgeranylation and guanine nucleotides. Cell Signal. 2018;44(44): 72-81.

21. Pocognoni CA, Berberián MV, Mayorga LS. ESCRT (Endosomal Sorting Complex Required for Transport) Machinery Is Essential for Acrosomal Exocytosis in Human Sperm. Biol Reprod. 2015;93(5):1-12.

22. de La Fuente JM, Berry CC. Tat peptide as an efficient molecule to translocate gold nanoparticles into the cell nucleus. Bioconjug Chem. 2005;16(5):1176-1180.

23. Sun L, Liu D, Wang Z. Functional gold nanoparticle-peptide complexes as cell-targeting agents. Langmuir. 2008;24(18):10293-10297.

24. Khamehchian S, Nikkhah M, Madani R, Hosseinkhani S. Enhanced and selective permeability of gold nanoparticles functionalized with cell penetrating peptide derived from maurocalcine animal toxin. J Biomed Mater Res A. 2016;104(11):2693-2700.
25. He B, Yang D, Qin M, et al. Increased cellular uptake of peptidemodified PEGylated gold nanoparticles. Biochem Biophys Res Commun. 2017:494(1-2):339-345.

26. Zong J, Cobb SL, Cameron NR. Peptide-functionalized gold nanoparticles: versatile biomaterials for diagnostic and therapeutic applications. Biomater Sci. 2017;5(5):872-886.

27. Quevedo MF, Lucchesi O, Bustos MA, Pocognoni CA, de La Iglesia PX, Tomes CN. The Rab3A-22A Chimera Prevents Sperm Exocytosis by Stabilizing Open Fusion Pores. J Biol Chem. 2016;291(44): 23101-23111.

28. Brewer SH, Glomm WR, Johnson MC, Knag MK, Franzen S. Probing BSA binding to citrate-coated gold nanoparticles and surfaces. Langmuir. 2005;21(20):9303-9307.

29. Haiss W, Thanh NT, Aveyard J, Fernig DG. Determination of size and concentration of gold nanoparticles from UV-vis spectra. Anal Chem. 2007;79(11):4215-4221. Available from: https://doi.org/10.1093/ humupd/dmp048.

30. World Health Organization. Department of Reproductive Health and Research WHO laboratory manual for the examination and processing of human semen. 5th edn. 2010.

31. Mendoza C, Carreras A, Moos J, Tesarik J. Distinction between true acrosome reaction and degenerative acrosome loss by a one-step staining method using Pisum sativum agglutinin. J Reprod Fertil. 1992;95(3):755-763.

32. Huang X, El-Sayed MA. Gold nanoparticles: Optical properties and implementations in cancer diagnosis and photothermal therapy. $J A d v$ Res. 2010;1(1):13-28.

33. Mosior M, Mclaughlin S. Electrostatics and reduction of dimensionality produce apparent cooperativity when basic peptides bind to acidic lipids in membranes. Biochim Biophys Acta. 1992;1105(1): 185-187.

34. Morris MC, Deshayes S, Heitz F, Divita G. Cell-penetrating peptides: from molecular mechanisms to therapeutics. Biol Cell. 2008;100(4):201-217.

35. Skrlj N, Drevenšek G, Hudoklin S, Romih R, Curin Erbec V, Dolinar M. Recombinant single-chain antibody with the Trojan peptide penetratin positioned in the linker region enables cargo transfer across the bloodbrain barrier. Appl Biochem Biotechnol. 2013;169(1):159-169.

36. Mercogliano CP, Derosier DJ. Concatenated metallothionein as a clonable gold label for electron microscopy. J Struct Biol. 2007; 160(1):70-82.

37. Diestra E, Fontana J, Guichard P, Marco S, Risco C. Visualization of proteins in intact cells with a clonable tag for electron microscopy. J Struct Biol. 2009;165(3):157-168.

38. Fernández de Castro I, Sanz-Sánchez L, Risco C. Metallothioneins for correlative light and electron microscopy. Methods Cell Biol. 2014;124(124):55-70.

39. Morphew MK, O’Toole ET, Page CL, et al. Metallothionein as a clonable tag for protein localization by electron microscopy of cells. J Microsc. 2015;260(1):20-29.

40. Pelletán LE, Suhaiman L, Vaquer CC, et al. ADP ribosylation factor 6 (ARF6) promotes acrosomal exocytosis by modulating lipid turnover and Rab3A activation. J Biol Chem. 2015;290(15):9823-9841.

41. Zanetti N, Mayorga LS. Acrosomal swelling and membrane docking are required for hybrid vesicle formation during the human sperm acrosome reaction. Biol Reprod. 2009;81(2):396-405.

42. Turkevich J, Garton G, Stevenson PC. The color of colloidal gold. J Colloid Sci. 1954;9(1):26-35.

43. Mercogliano CP, Derosier DJ. Gold nanocluster formation using metallothionein: mass spectrometry and electron microscopy. J Mol Biol. 2006;355(2):211-223.

44. Barchanski A, Taylor U, Sajti CL, et al. Bioconjugated Gold Nanoparticles Penetrate Into Spermatozoa Depending on Plasma Membrane Status. J Biomed Nanotechnol. 2015;11(9):1597-1607.

45. Taylor U, Barchanski A, Petersen S, et al. Gold nanoparticles interfere with sperm functionality by membrane adsorption without penetration. Nanotoxicology. 2014;8 Suppl 1(1):118-127. 


\section{Supplementary materials}

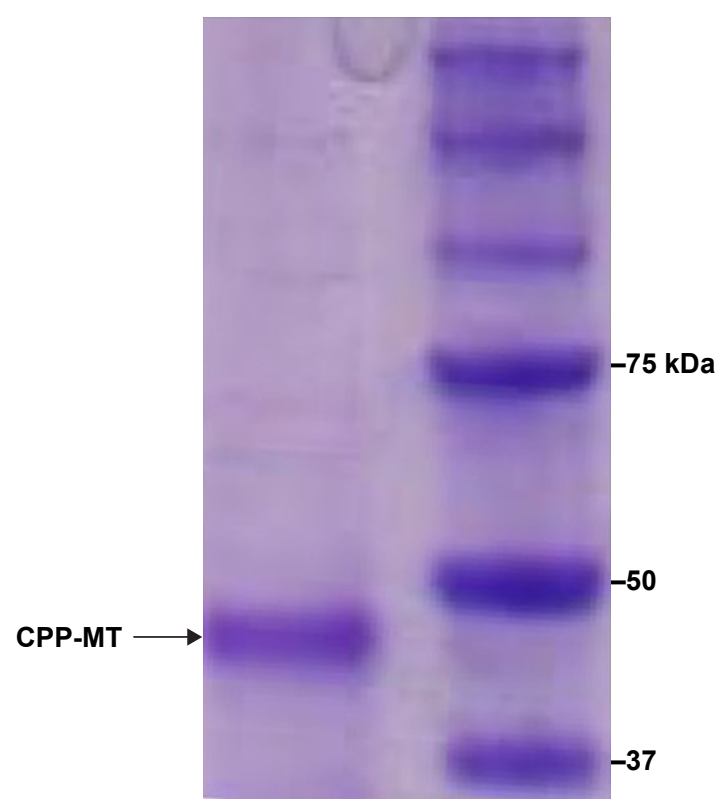

Figure SI SDS/PAGE of the CPP-MT recombinant protein.

Notes: A clear band is observed at $43 \mathrm{kDa}$, associated with the CPP-MT itself. Coomassie blue staining of the gel showed no evidence of bands associated with lipopolysaccharide contamination.

Abbreviations: CPP, cell-penetrating peptide; MT, metallothionein.

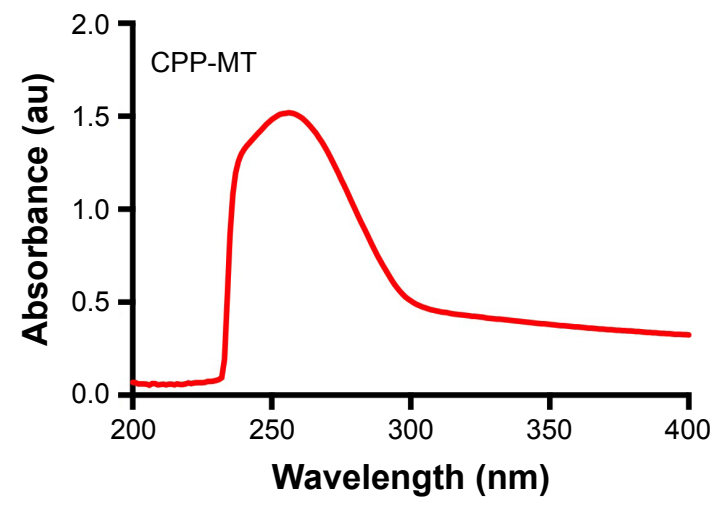

Figure S2 UV absorption spectrum of CPP-MT in phosphate buffer.

Notes: The spectrum shows no signs of lipopolysaccharide contamination. In fact, the absorption maximum occurs at $260 \mathrm{~nm}$ due to the small fraction of aromatic amino acids, which would even facilitate the identification of lipopolysaccharide by UV-Vis spectroscopy.

Abbreviations: CPP, cell-penetrating peptide; MT, metallothionein.
International Journal of Nanomedicine

\section{Publish your work in this journal}

The International Journal of Nanomedicine is an international, peerreviewed journal focusing on the application of nanotechnology in diagnostics, therapeutics, and drug delivery systems throughout the biomedical field. This journal is indexed on PubMed Central, MedLine, CAS, SciSearch $\AA$, Current Contents ${ }^{\circledR} /$ Clinical Medicine,

\section{Dovepress}

Journal Citation Reports/Science Edition, EMBase, Scopus and the Elsevier Bibliographic databases. The manuscript management system is completely online and includes a very quick and fair peer-review system, which is all easy to use. Visit http://www.dovepress.com/ testimonials.php to read real quotes from published authors. 